\title{
Design of a Single-Feed Dual-Band Dual-Polarized Printed Microstrip Antenna Using a Boolean Particle Swarm Optimization
}

\author{
Farzaneh Afshinmanesh, Student Member, IEEE, Alireza Marandi, Student Member, IEEE, and Mahmoud Shahabadi
}

\begin{abstract}
A novel dual-frequency dual-linear-polarization printed antenna element benefiting from a single-feed single-layer structure is introduced in this paper. The Boolean particle swarm optimization algorithm in conjunction with the method of moments (MoM) is employed to optimize the geometry of the antenna after considering three objectives: cross polarization, return loss, and boresight direction in both bands. A fuzzy-logic based ordered weighted averaging operator allows us to efficiently implement the multi-objective optimization technique. Prototypes of the optimized designs have been fabricated and tested. The measured results show excellent performance with more than $15 \mathrm{~dB}$ of return loss and $10 \mathrm{~dB}$ of cross polarization in both frequency bands of operation, i.e., 12 and $14 \mathrm{GHz}$. A gain of $4.8 \mathrm{dBi}$ has been measured for both frequency bands.
\end{abstract}

Index Terms-Advanced optimization techniques in electromagnetics, Boolean particle swarm optimization (BPSO), dual-band dual-polarized printed antenna, fuzzy logic.

\section{INTRODUCTION}

$\mathbf{P}$ RINTED antennas offer an attractive alternative to a number of antennas used in communications systems due to their lower profile, weight, and manufacturing costs, as well as their compatibility with integrated circuit technologies. An increasing need for enhancing the capabilities of wireless systems has led to various antenna designs with multiple functionalities among which one can refer to microstrip antennas that operate in dual frequency bands, or radiate different polarizations in different bands. Evidently, the complexity of antenna design increases with the number of operating frequency bands.

To realize dual frequency operation, a variety of methods has been proposed so far. Using slots in the patch [1], [2], loading the patch with shorting pins [3]-[5], using stacked patches [6]-[9], or using two feeding ports [9] are the mostly exploited ones. In addition, there are other planar antennas of special geometries to achieve dual-band operation. On the one hand, there are non-rectangular microstrip antennas loaded with

Manuscript received September 20, 2006; revised November 2, 2007. Published July 7, 2008 (projected).

F. Afshinmanesh and A. Marandi were with the School of Electrical and Computer Engineering, University of Tehran, Tehran 14395-15, Iran. They are now with the Department of Electrical and Computer Engineering, University of Victoria, Victoria, BC V8P 5C2, Canada (e-mail: farzaneh@ece.uvic.ca, marandi@ece.uvic.ca).

M. Shahabadi is with the School of Electrical and Computer Engineering, University of Tehran, Faculty of Engineering, Tehran, 14395-15, Iran (e-mail: shahabad@ut.ac.ir).

Color versions of one or more of the figures in this paper are available online at http://ieeexplore.ieee.org.

Digital Object Identifier 10.1109/TAP.2008.924684

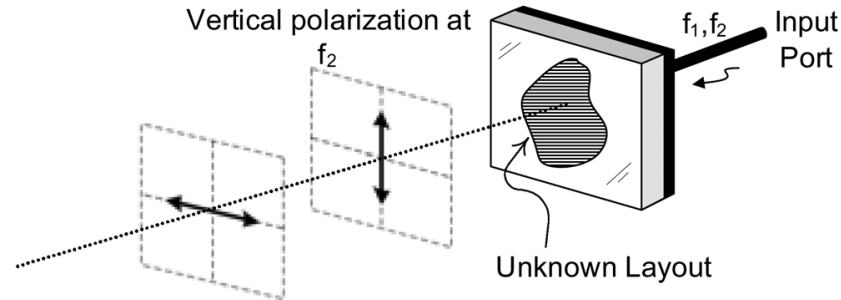

Horizontal polarization at

$f_{1}$

Fig. 1. Polarizations of the proposed antenna in two bands.

non-canonical slots[11], [12], and on the other, there are special layouts obtained by using evolutionary algorithms [13]-[15].

To realize dual-band dual-polarization operation, a number of researchers have used stacked patches with two separate feeds for each frequency band and polarization. In these structures, a combination of two different feeds is observed. These feeds are composed of two separate microstrip lines [16], two apertures [17], one via and one microstrip [18], two separate probes [19], and one microstrip and one aperture [20]. Other designs with a single electromagnetically coupled feed are also available [21], [22]. In general, stacked patches suffer from disadvantages such as thick substrate, difficult manufacturing, and high cost. On the other hand, using single feed antennas can reduce complexity and cost of the receiver front-end. Patch antennas with switchable slots (PASS) are single-layer single-feed versions of dual-band dual-polarized antennas [23]; however, because of switches, they cannot operate at very high frequencies.

The main goal of this work is to design and realize a dual-band and dual-polarized printed antenna that has a simple metallic layout and benefits from a single-feed, single-layer structure. In order to be applicable to a two-way satellite Internet system, the antenna should radiate a linearly polarized wave at $f_{1}=12 \mathrm{GHz}$ and a linearly polarized wave orthogonal to it at $f_{2}=14 \mathrm{GHz}$ as depicted in Fig. 1 .

Due to the large number of parameters involved in designing the metallic layout of such an antenna, we utilize an evolutionary computation technique called the Boolean particle swarm optimization (BPSO). It allows us to design the appropriate layout without any a priori knowledge of possible configurations. Being recently introduced to the EM community [24], the PSO algorithm has been found to be an effective alternative in handling different antenna design problems. Compared to other evolutionary algorithms like genetic algorithm, the PSO algorithm is easier to implement 


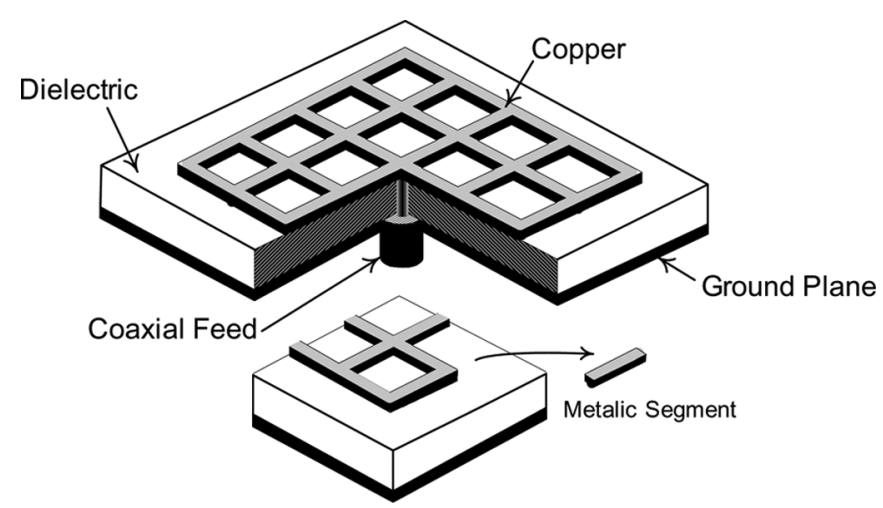

Fig. 2. Configuration of the planar antenna used in Boolean PSO design.

and to apply to various design problems with real-valued or discrete parameters. The Boolean PSO is a novel version of this algorithm in binary spaces and has been shown to be effective in dealing with antenna design problems [25].

The basic antenna configuration is a grid structure of metallic segments printed on the grounded substrate as shown in Fig. 2. The optimal layout will be obtained by removing some of the metallic segments. An electromagnetic solver based on the method of moments is used to predict the performance of each antenna designed by the Boolean PSO. In our approach, we take into account three objectives: cross polarization, return loss, and boresight direction in both frequency bands. In conjunction with the BPSO, a fuzzy-logic based ordered weighted averaging (OWA) operator is also used to facilitate designing the layout by implementing a transformation from the three-dimensional objective space to the one-dimensional cost function. To achieve a satisfactory result from the optimization process, different sets of BPSO parameters are explored. The optimized layout is obtained by applying the best found set of parameters. Lastly, the final design is obtained by trimming the optimized layout. This is then fabricated and tested.

The paper is organized as follows. In Section II, the details of the Boolean PSO approach is described. Section III describes the antenna parameters along with the objectives and the fuzzy OWA operator. Section IV describes the Boolean PSO design and its modifications as well as experimental verification of the results. Section $\mathrm{V}$ provides some concluding remarks.

\section{THE BOOLEAN PSO}

The Boolean particle swarm optimization is a new evolutionary computation technique implemented by means of the Boolean algebra to meet the requirements of a real-valued particle swarm optimization [25]. The real-valued PSO, introduced by Kennedy and Eberhart in 1995 [30], is initialized with a population of random solutions, called particles. Each particle moves through the search space with a velocity that is dynamically adjusted according to its own and its companion's previous behavior. Updating the particle velocity is based on three terms, namely the "social," the "cognitive," and the "inertia" terms. The "social" part is the term guiding the particle to the best position achieved by the whole swarm of particles so far $\left(g_{\text {best }}\right)$, the "cognitive" term conducts it to the best position achieved by itself so far $\left(p_{\text {best }}\right)$, and the "inertia" part is the memory of its previous velocity $\left(\omega \cdot v_{n}\right)$. The following formulae demonstrate the updating process of a particle position $\left(x_{n}\right)$ and its velocity $\left(v_{n}\right)$ in the $n$th dimension in an $N$-dimensional optimization:

$$
\begin{aligned}
v_{n}= & \omega \cdot v_{n}+c_{1} \cdot \phi_{1} \cdot\left(p_{\text {best }, n}-x_{n}\right) \\
& +c_{2} \cdot \phi_{2} \cdot\left(g_{\text {best }, n}-x_{n}\right) \\
x_{n}= & x_{n}+v_{n} .
\end{aligned}
$$

$\varphi_{1}$ and $\varphi_{2}$ are random numbers uniformly distributed between 0 and $1, c_{1}$ and $c_{2}$ are acceleration constants and $\omega$ is the inertia weight. These three parameters determine the tendency of the particles to the related terms. Moreover, by allowing a limit on the maximum velocity $\left(V_{\max }\right)$, the convergence rate of the optimization process can be controlled.

In the Boolean PSO, distance and velocity are defined based on the difference between corresponding bits of two binary strings. The "AND" $(\cdot)$, “OR" $(+)$, and "XOR" $(\oplus)$ operators are used to model movement of the particles. Main formulae for updating the $d$ th bit of position and velocity of each particle are

$$
\begin{aligned}
& v_{d}=\omega \cdot v_{d}+c_{1} \cdot\left(p_{\text {best }, d} \oplus x_{d}\right)+c_{2}\left(g_{\text {best }, d} \oplus x_{d}\right) \\
& x_{d}=x_{d} \oplus v_{d} .
\end{aligned}
$$

The distance between two bits is another bit whose value represents their difference. Consequently, the velocity bit, $v_{d}$, determines whether the $d$ th bit of the position of the particle, $x_{d}$, should change in the next step, according to (4). In (3), the second and third terms calculate the distance between $\left(x_{d}, p_{\text {best }, d}\right)$ and $\left(x_{d}, g_{\text {best }, d}\right)$, respectively. The three terms are then combined using "OR" operators. $c_{1}, c_{2}$ as the acceleration coefficients and $\omega$ as the inertia coefficient are binary bits stochastically set from the system parameters $C_{1}, C_{2}, \Omega$, acceleration constants and inertia weight, which are real numbers between 0 and 1 . The probabilities for being " 1 " of c1, c $2, \omega$ are $C_{1}, C_{2}$, and $\Omega$. The procedure used to specify the velocity for each bit of a particle is depicted in the schematic diagram of Fig. 3. In this procedure, $V_{\max }$ is the number of allowed " 1 " bits in the calculated velocity array. To prevent the particles from moving faster than this value, each calculated velocity array is examined for the number of " 1 ." If this number exceeds the desired value, one bit with a value of " 1 " is set to zero randomly, and this process continues until the satisfaction of the criterion.

The simplicity in implementation of the Boolean PSO and also the straightforward influence of its parameters on the optimization process are from its key benefits over the first version of the binary PSO introduced by Kennedy [33] and also genetic algorithm. Its superior performance over those methods in some cases has been shown in [25], [26].

\section{ANTENNA DESIGN}

In our approach, the Boolean PSO is used to facilitate designing the layout of a single-feed antenna that radiates two orthogonal linear polarizations at two different frequencies. For this purpose, the optimizer should search in the space of binary strings in which an antenna layout is represented by a string whose bits specify whether the corresponding metallic segments on the layout should be removed. In each step, after evaluating 


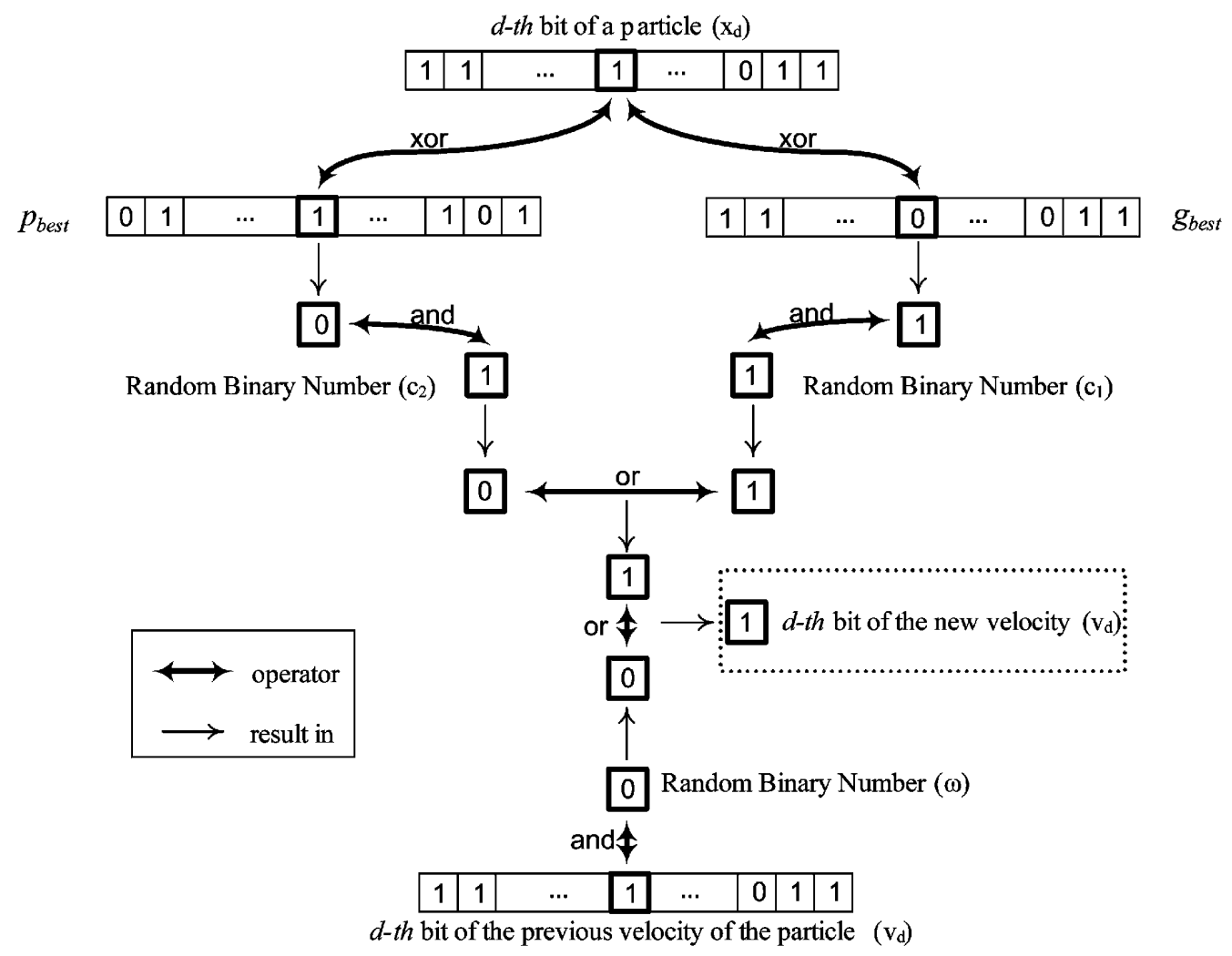

Fig. 3. Process of updating the velocity in the Boolean PSO.

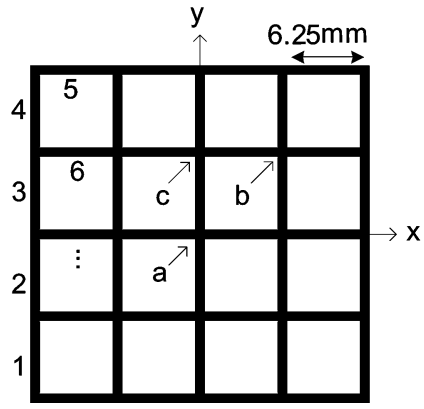

Fig. 4. Grid with possible feed positions (a, b, c). Also shown is the order of segments in the coded array.

the characteristics of the antenna by a MoM engine, its corresponding string receives a real number fitness which is calculated by an OWA according to three objectives derived from the antenna pattern, return loss, and gain.

For this work, the desired operation frequencies are $12 \mathrm{GHz}$ and $14 \mathrm{GHz}$. Fig. 4 shows the metallic grid from which the desired layout should be generated. Also shown in Fig. 4 is the coding order in the 40-bit coded array; the grid size is determined with respect to the working frequencies. As depicted in Fig. 4, due to the symmetry in the grid structure, there are three possible positions for placing the feed: $a, b$, and c. For determining the best feed position and because of the complexity of the multidimensional multi-objective problem, the optimization process is performed three times for these three possible positions, so the feed position is not included in the optimization vector. The metallic conductor is copper with a conductivity of $5.7 \times 10^{7} \mathrm{~S} / \mathrm{m}$, and the antenna substrate is RT/Duroid 5880 $\left(\varepsilon_{r}=2.2\right)$ with a thickness of $62 \mathrm{mil}$. The antenna is designed to match to $50 \Omega$, and an infinite conducting ground plane is assumed in the simulation carried out by the MoM solver. Moreover, the resolution of the MoM mesh is one-third of the segment size.

\section{A. Evaluation of Objectives}

Calculating the fitness of an antenna to the desired specifications is based on three design goals: orthogonal linear polarization at two different frequencies $f_{1}$ and $f_{2}$, small return loss, and high gain around $\theta=0^{\circ}$ in both bands. The three objectives associated with these design goals are: polarization objective $\left(o b j_{p o l}\right)$, return loss objective $\left(\mathrm{obj}_{S 11}\right)$, and gain objective $\left(\mathrm{obj}_{\mathrm{Gain}}\right)$. The real number assigned to a particle is calculated with an OWA operator equipped with a Mamdani fuzzy inference system, which is a transformation from three-dimensional objective space to the one-dimensional fitness space. An antenna with a smaller fitness value has a better performance and zero fitness means that the antenna fulfills all the design goals.

\section{B. Polarization Objective}

Here, the polarization objective, obj $j_{\text {pol }}$, stands for the amount of orthogonality of the polarizations at the two working frequencies. obj $j_{\text {pol }}=0$ shows that the antenna has two orthogonal linear polarizations at two frequencies of $f_{1}$ and $f_{2}$. To evaluate this objective, the criterion is the ratio of the magnitude of $E_{\theta}$ to $E_{\varphi}$, or vice versa, at all angles within $-\theta_{1} \leq \theta \leq \theta_{1}$ in both $\varphi=0^{\circ}$ and $\varphi=90^{\circ}$ planes. (Note that the above angles are defined in a spherical coordinate system superimposed on the 
coordinate system shown in Fig. 4.) This ratio must be less than a certain value MINP, to fulfill dual-linear-polarization design goal.

At each frequency and for an antenna, $E_{\theta}$ and $E_{\varphi}$ are computed in both $\varphi=0^{\circ}$ and $\varphi=90^{\circ}$ planes for $-\theta_{1} \leq \theta \leq \theta_{1}$ with $\theta_{1}=10^{\circ}$. Then the axial ratio (AR) is computed at each angle according to

$$
\begin{aligned}
& \operatorname{AR}_{1}(\theta)=\left|\frac{E_{\theta}}{E_{\varphi}}\right| \quad \phi_{1}=0^{\circ} \text { plane } \quad f_{1}=12 \mathrm{GHz} \\
& \mathrm{AR}_{2}(\theta)=\left|\frac{E_{\varphi}}{E_{\theta}}\right| \quad \phi_{2}=90^{\circ} \text { plane } f_{1}=12 \mathrm{GHz} \\
& \mathrm{AR}_{3}(\theta)=\left|\frac{E_{\theta}}{E_{\varphi}}\right| \quad \phi_{1}=0^{\circ} \text { plane } \quad f_{2}=14 \mathrm{GHz} \\
& \mathrm{AR}_{4}(\theta)=\left|\frac{E_{\varphi}}{E_{\theta}}\right| \quad \phi_{2}=90^{\circ} \text { plane } f_{2}=14 \mathrm{GHz} \text {. }
\end{aligned}
$$

It is necessary to have orthogonal polarizations at the working frequencies; however, the sense of polarization at each frequency is not important. The following process is done to specify the type of polarization which results in a better objective value:

$$
\begin{aligned}
& \operatorname{Ave}_{n}(\theta)=\frac{1}{2 \theta_{1}} \int_{\theta=-\theta_{1}}^{\theta_{1}}\left(\operatorname{AR}_{n}(\theta) d \theta\right) \quad n=1, \ldots, 4 \\
& \left\{\begin{array}{lll}
\text { if } & \text { Ave }_{1}+\text { Ave }_{2}>A v e_{3}+\text { Ave }_{4} \\
& \mathrm{AR}_{n}=\left(A R_{n}\right)^{-1} & n=1,2 \\
\text { else } & \mathrm{AR}_{n}=\left(\mathrm{AR}_{n}\right)^{-1} & n=3,4 .
\end{array}\right.
\end{aligned}
$$

The $g_{n}(\theta)$ function is defined to assign zero to the $\mathrm{obj}_{\mathrm{pol}}$ for axial ratios less than or equal to MINP with MINP $=-15 \mathrm{~dB}$, and a positive number for other values

$$
\begin{aligned}
g_{n}(\theta)= & \left\{d B\left[\operatorname{AR}_{n}(\theta)\right]-\operatorname{MINP}\right\} \\
& \cdot u\left\{d B\left[\operatorname{AR}_{n}(\theta)\right]-\operatorname{MINP}\right\} \\
n= & 1, \ldots, 4 .
\end{aligned}
$$

In (10), " $u$ " refers to the Heaviside step function.

Thus

$$
\operatorname{obj}_{\mathrm{pol}}=\frac{1}{4}\left(\sum_{n=1}^{4} \frac{1}{2 \theta_{1}} \int_{\theta=-\theta_{1}}^{\theta_{1}} g_{n}(\theta) d \theta\right) .
$$

Moreover, because of the fuzzification layer used in the OWA operator, an upper limit is needed for obj $\mathrm{pol}_{\mathrm{pol}}$, which is set to 15 . Therefore, if the calculated obj $j_{\text {pol }}>15$, then we set it to 15 . It is worth to mention that to calculate the integrals in (9) and (11), they are discretized with $\Delta \theta=1^{\circ}$ discretization step.

\section{Return Loss Objective}

The return loss objective, obj ${ }_{S 11}$, represents the amount of impedance matching at the two working frequencies. Similar to the polarization objective, this real number is between 0 and 15 , and its zero value shows the satisfied goal, that is to say a return loss of at least $-15 \mathrm{~dB}$ at both frequencies. In a mathematical expression

$\operatorname{obj}_{S 11}=\frac{1}{2} \sum_{n=1}^{2}\left\{\left.\mathrm{~dB}\left(S_{11}\right)\right|_{f_{n}}+15\right\} \cdot u\left\{\left.\mathrm{~dB}\left(S_{11}\right)\right|_{f_{n}}+15\right\}$

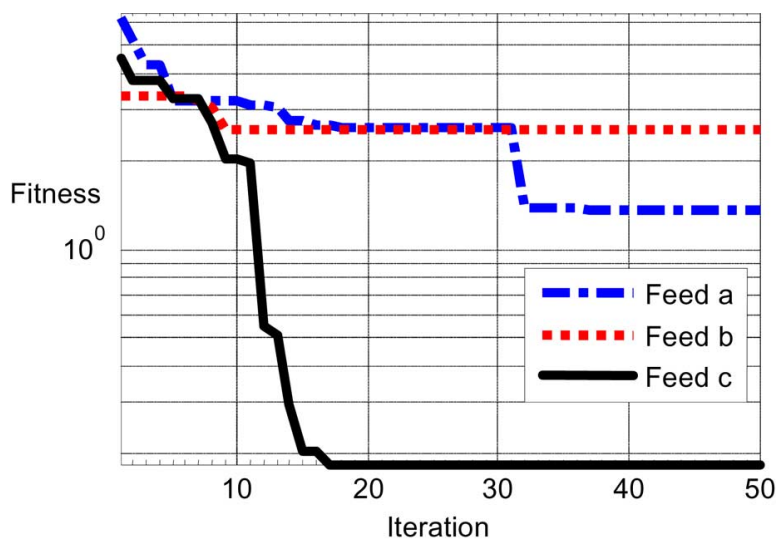

Fig. 5. Best fitness of iterations with three possible feed positions.

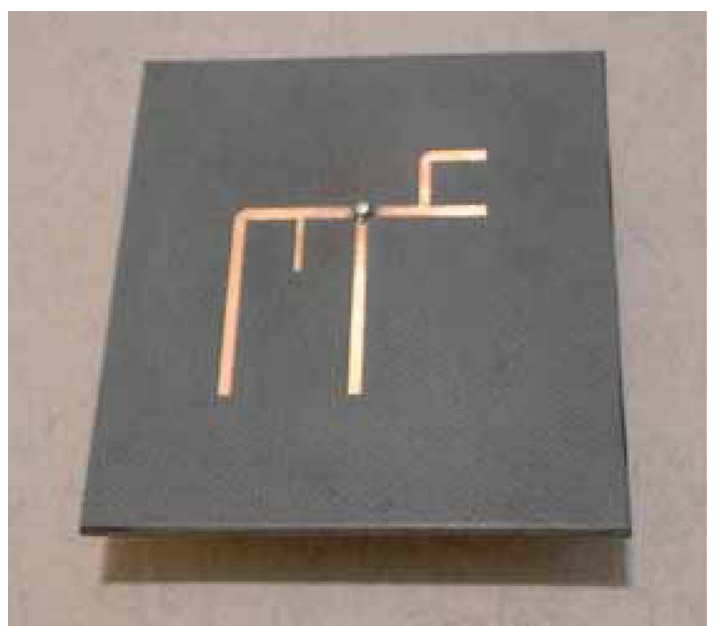

Fig. 6. Boolean PSO-designed antenna element fabricated on RT-Durroid 5880 .

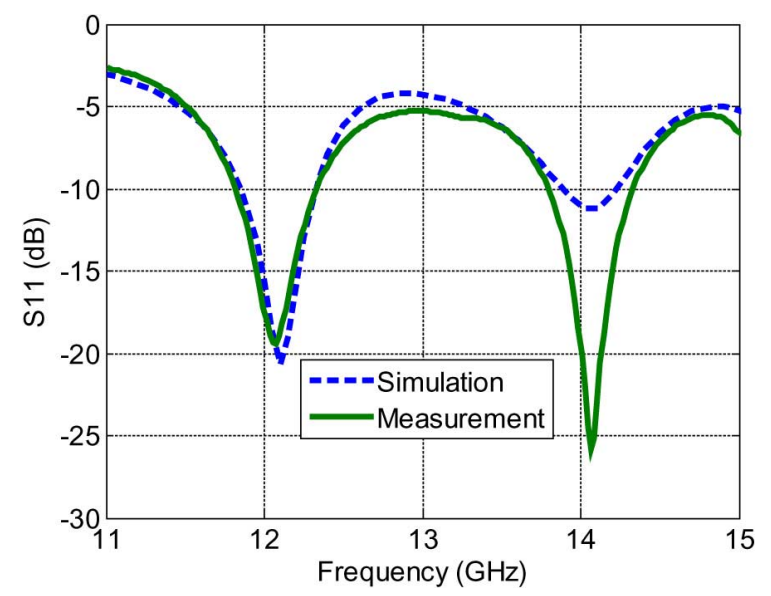

Fig. 7. Measured and simulated return loss of the Boolean-PSO designed antenna.

\section{Gain Objective}

This objective is defined to place the boresight around $\theta=0^{\circ}$. The gain objective, objain, is the average of differences between the maximum gain and the average gain in all planes at both frequencies, minus unity. $\mathrm{obj}_{\text {Gain }}=0$ means that in all 


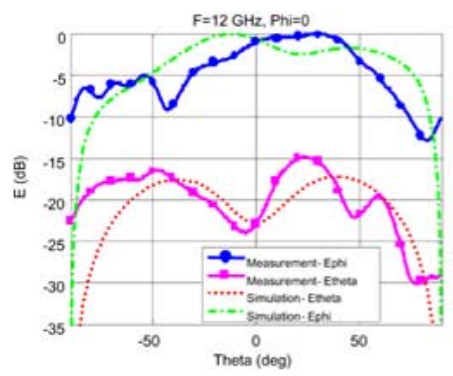

(a)

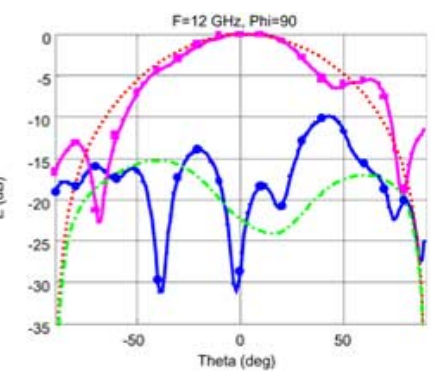

(b)

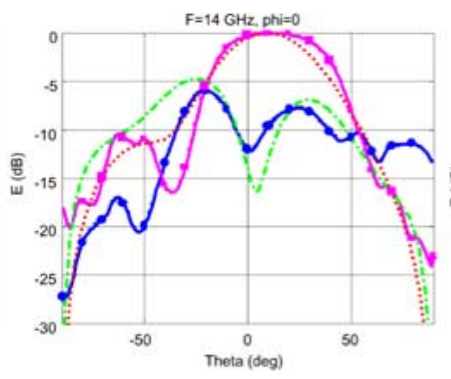

(c)

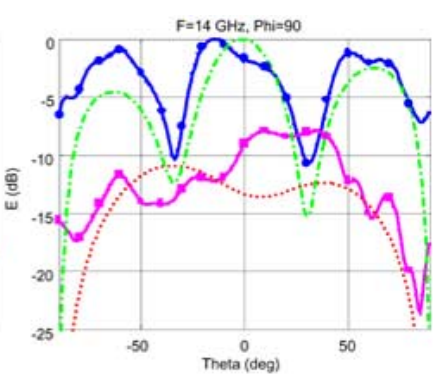

(d)

Fig. 8. Measured and simulated radiation patterns of the Boolean PSO designed antenna in different planes at operation frequencies, (a) $x-z$ plane at $12 \mathrm{GHz}$, (b) $y$-z plane at $12 \mathrm{GHz}$, (c) $x-z$ plane at $14 \mathrm{GHz}$, (d) $y-z$ plane at $14 \mathrm{GHz}$.

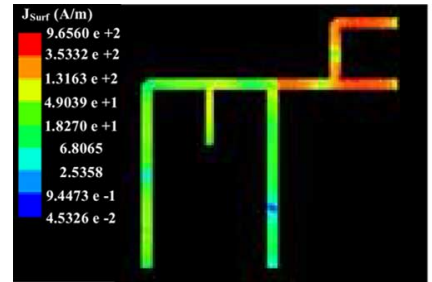

(a) $\mathrm{f}=12 \mathrm{GHz}$

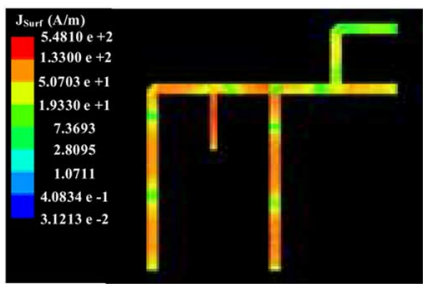

(b) $\mathrm{f}=14 \mathrm{GHz}$

Fig. 9. Current distribution on the Boolean PSO designed layout at operation frequencies.

planes and at both frequencies the difference between the maximum and average gain is less than $1 \mathrm{~dB}$. Like other objectives, this objective has an upper limit which is equal to 3 . This objective is given by

$$
\begin{aligned}
\operatorname{GMAX}\left(\varphi_{i}, f_{j}\right)= & \max \left\{\operatorname{gain}\left(\varphi_{i}, f_{j}, \theta\right)\right\} \\
i, j= & 1,2, \quad-90 \leq \theta \leq 90 \\
h_{n}\left(\varphi_{i}, f_{j}\right)= & \operatorname{GMAX}\left(\varphi_{i}, f_{j}\right) \\
& -\frac{1}{2 \theta_{1}}\left(\sum_{j=1}^{2} \sum_{i=1}^{2} \int_{-\theta_{1}}^{\theta_{1}} \operatorname{gain}\left(\varphi_{i}, f_{j}, \theta\right) d \theta\right) \\
\operatorname{obj}_{\text {Gain }}= & \left(\frac{1}{4} \sum_{j=1}^{2} \sum_{i=1}^{2} h_{n}\left(\varphi_{i}, f_{j}\right)\right)-1 .
\end{aligned}
$$

\section{E. Fuzzy OWA Operator for Multiobjective Optimization}

The transformation from three-dimensional objective space to a one-dimensional fitness space is performed with an OWA operator [27], a transformation of the objectives to be aggregated by their relative importance with an associated weighting vector

$$
W=\left[\begin{array}{l}
w_{1} \\
w_{2} \\
w_{3}
\end{array}\right]
$$

in which

$$
\begin{gathered}
w_{j} \in[0,1], \quad \sum_{j=1}^{3} w_{j}=1 \\
F\left(\mathrm{obj}_{\mathrm{pol}}, \mathrm{obj}_{S 11}, \mathrm{obj}_{\text {gain }}\right)=\sum_{j=1}^{3} w_{j} b_{j} .
\end{gathered}
$$

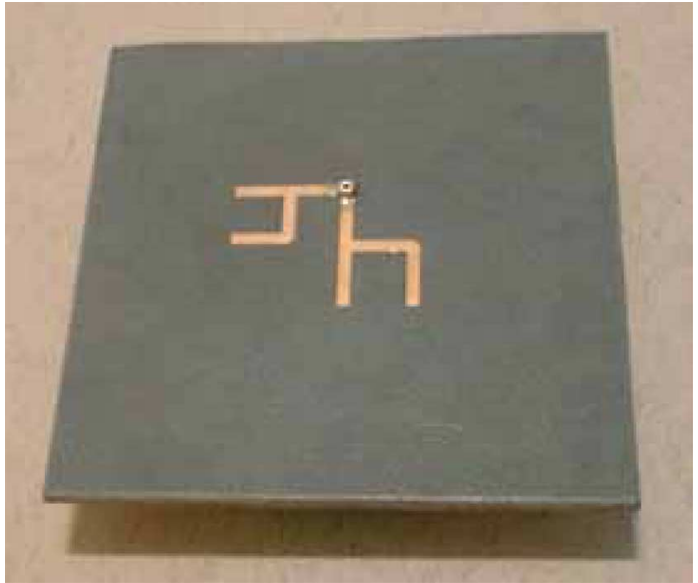

Fig. 10. Final antenna layout fabricated on RT/Duroid 5880.

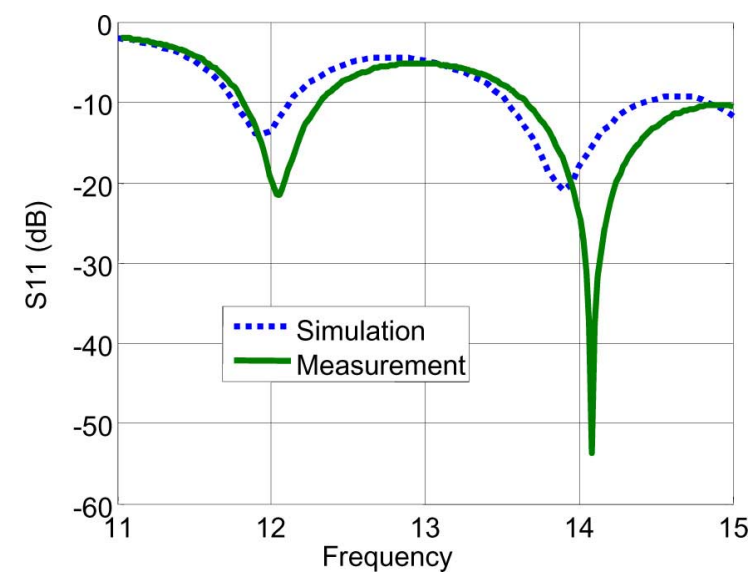

Fig. 11. Measured and simulated return loss of the final antenna.

The $\left[b_{1}, b_{2}, b_{3}\right]$ is the ordered form of $\left[\mathrm{obj}_{\mathrm{pol}}, \mathrm{obj}_{S 11}, \mathrm{obj}_{\mathrm{Gain}}\right]$ in which $b_{1}$ is the maximum, and $b_{3}$ is the minimum of the objectives. In addition to the mentioned characteristics, this operator should be commutative, monotonic, and idempotent.

As it is shown in (18), this operator calculates a linear weighted summation of all objective values, however, in this method the weights for a set of objectives are determined dynamically by a fuzzy inference system. All three objectives are normalized according to their ranges and the worst objective has the highest effect on the fitness. The fuzzification layer of the fuzzy inference system specifies how bad an objective 


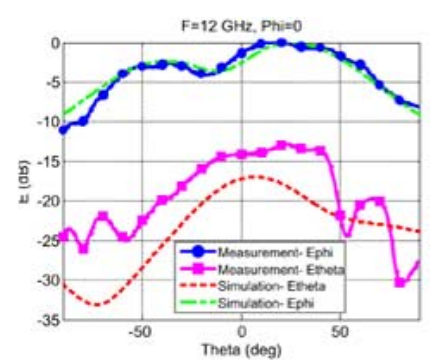

(a)

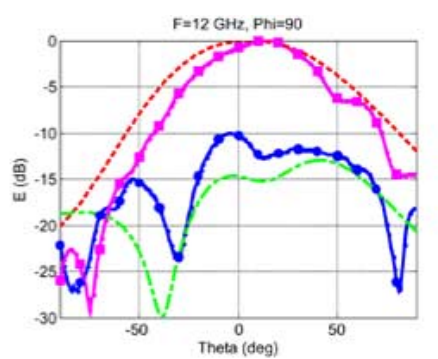

(b)

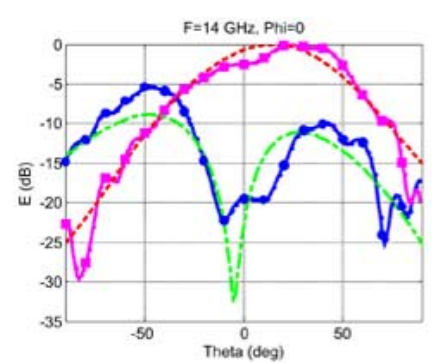

(c)

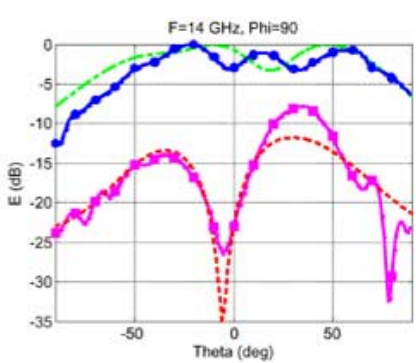

(d)

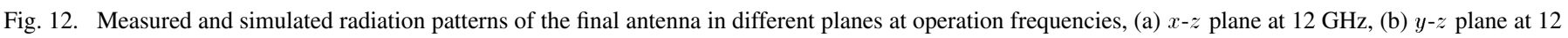
$\mathrm{GHz}$, (c) $x-z$ plane at $14 \mathrm{GHz}$, (d) $y-z$ plane at $14 \mathrm{GHz}$.

is in regard to other objectives, and the defuzzification layer calculates the weights based on that.

A Mamdani fuzzy inference system [28] which has three Gaussian membership functions for fuzzification layer is applied to the normalized objectives. The defuzzification layer contains similar membership functions and creates $W$ vector with specified characteristics. More details about combination of OWA and fuzzy logic can be found in [29].

\section{RESULTS}

\section{A. Boolean PSO-Designed Antenna}

For implementing the optimization process, the MATLAB [32] is used as the programming environment and an electromagnetic solver based on the method of moments as the antenna simulator. Because each antenna simulation takes about 30 seconds on a Pentium IV computer with 512 MB of RAM, the whole process is very time consuming. Therefore, only three different sets of parameters $(\Omega, \mathrm{C} 1, \mathrm{C} 2, \mathrm{~V} \max )$ are used for running the algorithm for each possible feed position. Fig. 5 shows the best obtained result for each feed position in 50 iterations with the population size of 400 . Consequently, feed c, depicted in Fig. 4, is chosen as the feed position that its corresponding parameters are $\Omega=0.1, C 1=C 2=0.5, \mathrm{Vmax}=15$. More details on how to determine the ideal parameters for PSO implementation can be found in [34], [35].

Fig. 6 shows the Boolean-PSO optimized design after being fabricated. The dimension of the ground plane is $50 \times 50 \mathrm{~mm}^{2}$. The simulated and measured return loss $\left(S_{11}\right)$ of the antenna is plotted in Fig. 7. The measurements have been carried out with an Agilent 8510C network analyzer. Measured return loss shows dual-band operation at the desired frequencies. Moreover, good agreement can be observed between the measurement and simulation.

Dual-band dual-polarization operation can be seen obviously in the simulated and measured radiation pattern plots in Fig. 8(a)-(d). In these figures, a commercial finite element package [31] is used to obtain the simulation results. We observe that at $12 \mathrm{GHz}$ [Fig. 8(a) and (b)] $E_{\varphi}$ is dominant in $\varphi=0^{\circ}$ plane and $E_{\theta}$ is dominant in $\varphi=90^{\circ}$ plane, whereas at $14 \mathrm{GHz}$ [Fig. 8(c) and (d)] a reverse condition exists. The antenna is optimized from $\theta=-10^{\circ}$ to $\theta=10^{\circ}$ and accordingly the radiation patterns satisfy the requirements in this range. Fig. 9(a) and (b) depicts the current distribution at $12 \mathrm{GHz}$ and $14 \mathrm{GHz}$, respectively. At $12 \mathrm{GHz}$ current flows predominantly

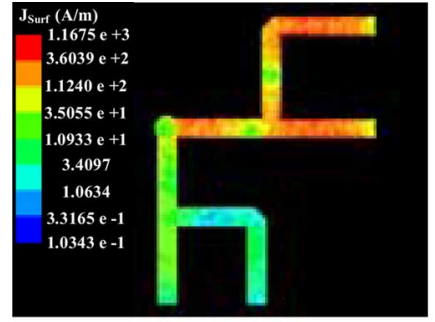

(a) $\mathrm{f}=12 \mathrm{GHz}$

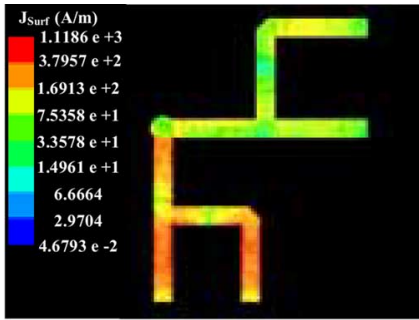

(b) $\mathrm{f}=14 \mathrm{GHz}$
Fig. 13. Current distribution on the modified design at operation frequencies.

in the pin-hair structure, while at $14 \mathrm{GHz}$, current flows mostly in the rotated E-shaped structure. The currents corresponding to the two bands are nearly mutually perpendicular. Note that without major mutual coupling, the mentioned two structures of the layout are separately responsible for the vertical and horizontal polarizations.

\section{B. Final Design}

Radiation patterns of the optimized element show better performance at all theta angles and at lower frequencies. As shown in the last section, the $12 \mathrm{GHz}$ band is mainly radiated by the horizontal pin-hair. Moreover, most of the antenna layouts obtained at local optima during the optimization process have this pin-hair as a part of their structure. Accordingly, we scale the pin-hair sub-structure to $14 \mathrm{GHz}$ and make use of it in place of the $14 \mathrm{GHz}$ E-shaped layout of Fig. 6. The new layout is shown in Fig. 10 which illustrates the fabricated antenna on RT/Duroid 5880. Obviously, the layout obtained in this fashion cannot be a member of the search space defined by the codes of Fig. 4. Return loss, radiation patterns, and current distribution of the modified element are shown in Figs. 11-13. In comparison with the previous structure, this antenna has appropriate radiation patterns in all theta angles in addition to a better return loss. The measured gain of the element is $4.8 \mathrm{dBi}$. The cross-polarization level is below $-10 \mathrm{~dB}$ at $11.8-12.2 \mathrm{GHz}$ and $13.6-14.1 \mathrm{GHz}$.

\section{CONCLUSION}

Realization of a single-feed dual-band and dual-polarized printed antenna has been demonstrated in this paper. Benefiting from a single-layer structure, the introduced antenna has a matching with more than $15 \mathrm{~dB}$ return loss in both bands. The cross polarization level is more than $10 \mathrm{~dB}$, and the antenna gain is about $4.8 \mathrm{dBi}$ in both bands. Design of the proposed antenna 
is carried out with the Boolean particle swarm optimization, a novel representation of the PSO algorithm in the binary space, which facilitates the design of such an antenna with many parameters involved. Recognizing the main radiating substructures of the optimized antenna, we have obtained an additional antenna layout which is not a member of the search space. The optimization procedure in this work is based on three objectives: cross polarization, return loss, and boresight direction in both bands, but more parameters can be added to the optimization process. An array of the designed antenna can be exploited in two-way satellite Internet systems.

\section{ACKNOWLEDGMENT}

The authors would like to thank the Center of Excellence on Applied Electromagnetic Systems at the University of Tehran for their invaluable support. The authors would also like to acknowledge the help of Dr. D. Busuioc at the University of Waterloo, Canada, for performing HFSS simulations.

\section{REFERENCES}

[1] S. Maci, G. B. Gentili, P. Piazzesi, and C. Salvador, "Dual band slot loaded patch antenna," Proc. Inst. Elect. Eng. Microw. Antennas Propag., vol. 142, pp. 225-232, Jun. 1995.

[2] B. F. Wang and Y. T. Lo, "Microstrip antennas for dual-frequency operation," IEEE Trans. Antennas Propag., vol. 32, pp. 938-943, Sep. 1984.

[3] C. L. Tang, H. T. Chen, and K. L. Wong, "Small circular microstrip antenna with dual-frequency operation," IEEE Electron. Lett., vol. 33, no. 13, pp. 1112-1113, Jun. 1997.

[4] K. L. Wong and W. S. Chen, "Compact microstrip antenna with dualfrequency operation," IEEE Electron. Lett., vol. 33, no. 8, pp. 646-647, Apr. 1997.

[5] S. C. Pan and K. L. Wand, "Dual frequency triangular microstrip antenna with shorting pin," IEEE Trans. Antennas Propag., vol. 45, pp. 1889-1891, Dec. 1997.

[6] L. Zaid, G. Kossiavas, J. Y. Dauvignac, J. Cazajous, and A. Papiemik, "Dual-frequency and broadband antennas with stacked quarter wavelength elements," IEEE Trans. Antennas Propag., vol. 47, no. 4, pp. 654-660, Apr. 1999.

[7] J. S. Dahele, K. F. Lee, and D. P. Wong, "Dual frequency stacked annular ring microstrip antenna," IEEE Trans. Antennas Propag., vol. 35, no. 11, pp. 1281-1285, Nov. 1987.

[8] F. Croq and D. M. Pozar, "Multifrequency operation on microstrip antennas using aperture coupled parallel resonators," IEEE Trans. Antennas Propag., vol. 40, no. 11, pp. 1367-1374, Nov. 1992.

[9] J. Wang, R. Fralich, C. Wu, and J. Litva, "Multifunctional aperture coupled stack patch antenna," IEEE Electron. Lett., vol. 26, no. 25, pp. 2067-2068, Dec. 1990.

[10] J. F. Zurcher, A. Skrivervik, O. Staub, and S. Vaccaro, "A compact dual-port dual-frequency printed antenna with high decoupling," $M i$ crow. Opt. Technol. Lett., vol. 19, pp. 131-137, Oct. 1998.

[11] K. L. Wong, M. C. Pan, and W. H. Hsu, "Single-feed dual-frequency triangular microstrip antenna with a v-shaped slot," Microw. Opt. Technol. Lett., vol. 20, pp. 133-134, Jan. 1999.

[12] J. Y. Jan, "Single-layer single-feed dual-frequency circular microstrip antenna with an offset open-ring slot," IEEE Trans. Antennas Propag., vol. 51, pp. 3010-3012, Oct. 2003.

[13] N. Jin and Y. Rahmat-Samii, "Parallel particle swarm optimization and finite- difference time-domain (PSO/FDTD) algorithm for multiband and wide-band patch antenna designs," IEEE Trans. Antennas Propag., vol. 53, no. 11, pp. 3459-3468, Nov. 2005.

[14] F. J. Villegas, T. Cwik, Y. Rahmat-Samii, and M. Manteghi, "A parallel electromagnetic genetic-algorithm optimization (EGO) application for patch antenna design," IEEE Trans. Antennas Propag., vol. 52, no. 9, pp. 2424-2435, Sep. 2004.

[15] H. Choo and H. Ling, "Design of broadband and dual-band microstrip antennas on a high-dielectric substrate using a genetic algorithm," in Proc. Inst. Elect. Eng. Microw. Antennas Propag., Jun. 2003, vol. 150, pp. 137-142.
[16] H. S. Noh, J. S. Yun, J. M. Kim, and S. I. Jeon, "Microstrip patch array antenna with high gain and wideband for Tx/Rx dual operation at Ku-band," in Proc. IEEE Antennas Propagation Society Int. Symp. Digest, Jun. 2004, vol. 3, pp. 2480-2483.

[17] N. C. Karmakar, S. Padhi, and S. Aditya, "Development of a portable VSAT antenna array for satellite communications," in Proc. IEEE AsiaPacific Microw. Conf., Dec. 2000, pp. 34-37.

[18] Y. Kim, W. Yun, and Y. Yoon, "Dual-frequency and dual-polarization wideband microstrip antenna," IEEE Electron. Lett., vol. 35, no. 17, pp. 1399-1400, Aug. 1999.

[19] K. S. Kona and Y. Rahmat-Samii, "Design and analysis of a novel probe-feeding method for stacked microstrip patch antennas," in IEEE Antennas Propagation Society Int. Symp. Digest, Jun. 2003, vol. 1, pp. $425-428$.

[20] J. S. Yun, H. S. Noh, S. I. Jeon, and C. J. Kim, "Design and analysis of $\mathrm{Tx} / \mathrm{Rx}$ dual microstrip antenna using FDTD at Ku-band," IEEE Electron. Lett., vol. 39, no. 17, pp. 1228-1230, Aug. 2003.

[21] S. V. Shynu, R. K. Raj, A. R. Chandran, C. K. Aanandan, P. Mohanan, and K. Vasudevan, "Single-feed dual-frequency dual polarized microstrip antenna with hexagonal slot," in IEEE Antennas Propagation Society Int. Symp. Digest, Jun. 2004, vol. 4, pp. 4380-4383.

[22] M. Paulson, S. O. Kundukulam, C. K. Aanandan, and P. Mohanan, "Compact arrow shaped antenna with embedded rectangular slot for dual frequency dual polarisation operation," in 2002 IEEE Antennas Propagat. Society Int. Symp. Digest, Jun. 2002, vol. 2, pp. 56-59.

[23] N. Jin, F. Yang, and Y. Rahmat-Samii, "A novel reconfigurable patch antenna with both frequency and polarization diversities for wireless communications," in IEEE Antennas ropagation Society Int. Symp. Digest, Jun. 2004, vol. 2, pp. 1796-1799.

[24] G. Ciuprina, D. Ioan, and I. Munteanu, "Use of intelligent-particle swarm optimization in electromagnetics," IEEE Trans. Magn., vol. 38 , pp. 1037-1040, Mar. 2002

[25] A. Marandi, F. Afshinmanesh, M. Shahabadi, and F. Bahrami, "Boolean particle swarm optimization and its application to the design of a dual-band dual-polarized printed antenna," in Proc. IEEE Congress Evolutionary Computation, Jul. 2006, pp. 3212-3218.

[26] F. Afshinmanesh, A. Marandi, and A. Rahimi-Kian, "A novel binary particle swarm optimization method using artificial immune system," in Proc. IEEE Int. Conf. on Computer as a Tool (EUROCON 2005), Nov. 2005, pp. 217-220.

[27] R. R. Yager, "On ordered weighted averaging aggregation operators in multicriteria decision making," IEEE Trans. Syst., Man Cybern., vol. 18, no. 1, pp. 183-190, Jan.-Feb. 1988.

[28] T. J. Ross, Fuzzy Logic with Engineering Applications, 2nd ed. New York: Wiley, 2004.

[29] R. R. Yager, "Including importances in OWA aggregations using fuzzy systems modeling," IEEE Trans. Fuzzy Syst., vol. 6, no. 2, pp. 286-294, May 1998.

[30] J. Kennedy and R. Eberhurt, "Particle swarm optimization," in Proc. IEEE Int. Conf. on Neural Networks, Nov.-Dec. 27-1, 1995, vol. 4, pp. 1942-1948.

[31] HFSS Version 9.0 Ansoft Corporation, Pittsburg, PA.

[32] MATLAB Version 7.0 The Mathworks Inc, Natick, MA.

[33] J. Kennedy and R. Eberhart, "A discrete binary version of the particle swarm algorithm," in Proc. IEEE Int. Conf. Systems, Man, Cybernetics, Oct. 1997, vol. 5, pp. 4104-4108.

[34] R. C. Eberhart and Y. Shi, "Particle swarm optimization: Developments, applications and resources," in Proc. IEEE Congress Evolutionary Computation, May 2001, vol. 1, pp. 81-86.

[35] J. Robinson and Y. Rahmat-Samii, "Particle swarm optimization in electromagnetics," IEEE Trans. Antennas Propag., vol. 52, no. 2, pp. 397-407, Feb. 2004

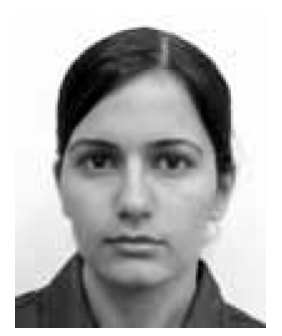

Farzaneh Afshinmanesh (S'03) received the B.Sc degree in electrical engineering from the University of Tehran, Tehran, Iran, in 2006. She is currently working toward the Master's degree at the University of Victoria, Victoria, BC, Canada.

From 2005 to 2006, she was a Research Assistant with the Center of Excellence on Applied Electromagnetic Systems, University of Tehran. Since September 2006, she has been working as a Graduate Research Assistant in the Computational Electromagnetics Research Laboratory and the Optical Systems and Technology Laboratory at the University of Victoria. Her current research interests are optoelectronics and photonic crystals. 


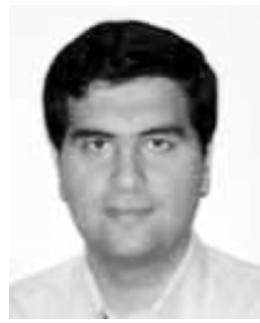

electromagnetics.

Mr. Marandi was the recipient of the Charles S. Humphrey Graduate Student Award from the University of Victoria in 2007.
Alireza Marandi (S'03) received the B.Sc. degree in electrical engineering from the University of Tehran, Tehran, Iran, in 2006. He is currently working toward the Master's degree at the University of Victoria, Victoria, BC, Canada.

He is a Research Assistant in the Optical Systems and Technology Laboratory and the Computational Electromagnetics Research Laboratory at the University of Victoria where he works on terahertz generation, nonlinear optics, optical and terahertz waveguiding and time-domain computational

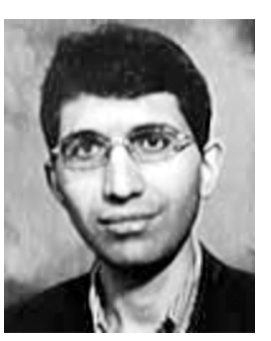

Mahmoud Shahabadi received the B.Sc. and M.Sc. degrees from the University of Tehran, Tehran, Iran, and the Ph.D. degree from Technische Universitaet Hamburg-Harburg, Germany, in 1988, 1991, and 1998, respectively, all in electrical engineering.

Since 1998, he has been an Assistant Professor and then an Associate Professor with the School of Electrical and Computer Engineering, University of Tehran. From 2001 to 2004, he was with the Department of Electrical and Computer Engineering, University of Waterloo, Canada, as a Visiting Professor. Additionally he is a co-founder and CTO of MASSolutions Inc., a Waterloo-based company with a focus on advanced low-profile antenna array systems. His research interests and activities encompass various areas of microwave and millimeter-wave engineering as well as photonics. Computational electromagnetics for microwave engineering and photonics are his special interest. He is currently conducting research and industrial projects in the field of antenna engineering, THz engineering, photonic crystals, plasmonics, left-handed materials, and holography.

Dr. Shahabadi was awarded the 1998/1999 Prize of the German Metal and Electrical Industries, Nordmetall, for his contribution to the field of millimeterwave holography and spatial power combining. 